DESY $96-215$

ISSN $0418-9833$

October 1996

\title{
Measurement of Charged Particle Transverse Momentum Spectra in Deep Inelastic Scattering
}

\author{
H1 Collaboration
}

\begin{abstract}
Transverse momentum spectra of charged particles produced in deep inelastic scattering are measured as a function of the kinematic variables $x$ and $Q^{2}$ using the H1 detector at the ep collider HERA. The data are compared to different parton emission models, either with or without ordering of the emissions in transverse momentum. The data provide evidence for a relatively large amount of parton radiation between the current and the remnant systems.
\end{abstract}




\section{H1 Collaboration}

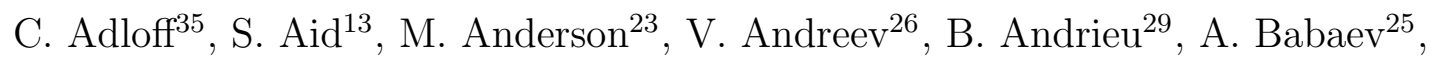
J. Bähr ${ }^{36}$, J. Bán ${ }^{18}$, Y. Ban ${ }^{28}$, P. Baranov ${ }^{26}$, E. Barrelet ${ }^{30}$, R. Barschke ${ }^{11}$, W. Bartel ${ }^{11}$, M. Barth ${ }^{4}$, U. Bassler ${ }^{30}$, H.P. Beck ${ }^{38}$, M. Beck ${ }^{14}$, H.-J. Behrend ${ }^{11}$, A. Belousov ${ }^{26}$, Ch. Berger ${ }^{1}$, G. Bernardi ${ }^{30}$, G. Bertrand-Coremans ${ }^{4}$, M. Besançon ${ }^{9}$, R. Beyer ${ }^{11}$, P. Biddulph ${ }^{23}$, P. Bispham ${ }^{23}$, J.C. Bizot ${ }^{28}$, V. Blobel ${ }^{13}$, K. Borras ${ }^{8}$, F. Botterweck ${ }^{27}$, V. Boudry ${ }^{29}$, A. Braemer ${ }^{15}$, W. Braunschweig', V. Brisson ${ }^{28}$, W. Brückner ${ }^{14}$, P. Bruel ${ }^{29}$, D. Bruncko ${ }^{18}$, C. Brune ${ }^{16}$, R. Buchholz ${ }^{11}$, L. Büngener ${ }^{13}$, J. Bürger ${ }^{11}$, F.W. Büsser ${ }^{13}$, A. Buniatian ${ }^{4}$, S. Burke ${ }^{19}$, M.J. Burton ${ }^{23}$, D. Calvet ${ }^{24}$, A.J. Campbell ${ }^{11}$, T. Carli ${ }^{27}$, M. Charlet ${ }^{11}$, D. Clarke $^{5}$, A.B. Clegg ${ }^{19}$, B. Clerbaux ${ }^{4}$, S. Cocks ${ }^{20}$, J.G. Contreras ${ }^{8}$, C. Cormack $^{20}$, J.A. Coughlan ${ }^{5}$, A. Courau ${ }^{28}$, M.-C. Cousinou ${ }^{24}$, G. Cozzika ${ }^{9}$, L. Criegee $^{11}$, D.G. Cussans ${ }^{5}$, J. Cvach $^{31}$, S. Dagoret ${ }^{30}$, J.B. Dainton ${ }^{20}$, W.D. Dau ${ }^{17}$, K. Daum ${ }^{42}$, M. David ${ }^{9}$, C.L. Davis ${ }^{19,39}$, B. Delcourt ${ }^{28}$, A. De Roeck ${ }^{11}$, E.A. De Wolf ${ }^{4}$, M. Dirkmann ${ }^{8}$, P. Dixon ${ }^{19}$, P. Di Nezza ${ }^{33}$, W. Dlugosz ${ }^{7}$, C. Dollfus ${ }^{38}$, K.T. Donovan ${ }^{21}$, J.D. Dowell ${ }^{3}$, H.B. Dreis ${ }^{2}$, A. Droutskoi ${ }^{25}$, O. Dünger ${ }^{13}$, H. Duhm ${ }^{12, \dagger}$ J. Ebert $^{35}$, T.R. Ebert ${ }^{20}$, G. Eckerlin ${ }^{11}$, V. Efremenko ${ }^{25}$, S. Egli ${ }^{38}$, R. Eichler ${ }^{37}$, F. Eisele ${ }^{15}$, E. Eisenhandler ${ }^{21}$, E. Elsen ${ }^{11}$, M. Erdmann ${ }^{15}$, W. Erdmann ${ }^{37}$, A.B. Fahr ${ }^{13}$, L. Favart ${ }^{28}$, A. Fedotov ${ }^{25}$, R. Felst ${ }^{11}$, J. Feltesse ${ }^{9}$, J. Ferencei ${ }^{18}$, F. Ferrarotto ${ }^{33}$, K. Flamm ${ }^{11}$, M. Fleischer ${ }^{8}$, M. Flieser ${ }^{27}$, G. Flügge ${ }^{2}$, A. Fomenko ${ }^{26}$, J. Formánek ${ }^{32}$, J.M. Foster ${ }^{23}$, G. Franke ${ }^{11}$, E. Fretwurst ${ }^{12}$, E. Gabathuler ${ }^{20}$, K. Gabathuler ${ }^{34}$, F. Gaede ${ }^{27}$, J. Garvey ${ }^{3}$, J. Gayler ${ }^{11}$, M. Gebauer ${ }^{36}$, H. Genzel ${ }^{1}$, R. Gerhards ${ }^{11}$, A. Glazov ${ }^{36}$, L. Goerlich ${ }^{6}$, N. Gogitidze ${ }^{26}$, M. Goldberg ${ }^{30}$, D. Goldner ${ }^{8}$, K. Golec-Biernat ${ }^{6}$, B. Gonzalez-Pineiro ${ }^{30}$, I. Gorelov ${ }^{25}$, C. Grab ${ }^{37}$, H. Grässler ${ }^{2}$, T. Greenshaw ${ }^{20}$, R.K. Griffiths ${ }^{21}$, G. Grindhammer ${ }^{27}$, A. Gruber ${ }^{27}$, C. Gruber ${ }^{17}$, T. Hadig ${ }^{1}$, D. Haidt ${ }^{11}$, L. Hajduk ${ }^{6}$, T. Haller ${ }^{14}$, M. Hampel ${ }^{1}$, W.J. Haynes ${ }^{5}$, B. Heinemann ${ }^{13}$, G. Heinzelmann ${ }^{13}$, R.C.W. Henderson ${ }^{19}$, H. Henschel ${ }^{36}$, I. Herynek ${ }^{31}$, M.F. Hess ${ }^{27}$, K. Hewitt ${ }^{3}$, W. Hildesheim ${ }^{11}$, K.H. Hiller ${ }^{36}$, C.D. Hilton ${ }^{23}$, J. Hladký ${ }^{31}$, M. Höppner ${ }^{8}$, D. Hoffmann ${ }^{11}$, T. Holtom ${ }^{20}$, R. Horisberger ${ }^{34}$, V.L. Hudgson ${ }^{3}$, M. Hütte ${ }^{8}$, M. Ibbotson ${ }^{23}$, H. Itterbeck ${ }^{1}$, A. Jacholkowska ${ }^{28}$, C. Jacobsson ${ }^{22}$, M. Jaffre ${ }^{28}$, J. Janoth ${ }^{16}$, D.M. Jansen ${ }^{14}$, T. Jansen ${ }^{11}$, L. Jönsson ${ }^{22}$, D.P. Johnson ${ }^{4}$, H. Jung ${ }^{22}$, P.I.P. Kalmus ${ }^{21}$, M. Kander ${ }^{11}$, D. Kant ${ }^{21}$, R. Kaschowitz ${ }^{2}$, U. Kathage ${ }^{17}$, J. Katzy ${ }^{15}$, H.H. Kaufmann ${ }^{36}$, O. Kaufmann ${ }^{15}$, M. Kausch ${ }^{11}$, S. Kazarian ${ }^{11}$, I.R. Kenyon ${ }^{3}$, S. Kermiche ${ }^{24}$, C. Keuker ${ }^{1}$, C. Kiesling ${ }^{27}$, M. Klein ${ }^{36}$, C. Kleinwort ${ }^{11}$, G. Knies ${ }^{11}$, T. Köhler ${ }^{1}$, J.H. Köhne ${ }^{27}$, H. Kolanoski ${ }^{36,41}$, S.D. Kolya ${ }^{23}$, V. Korbel ${ }^{11}$, P. Kostka ${ }^{36}$, S.K. Kotelnikov ${ }^{26}$, T. Krämerkämper ${ }^{8}$, M.W. Krasny ${ }^{6,30}$, H. Krehbiel ${ }^{11}$, D. Krücker ${ }^{27}$, H. Küster ${ }^{22}$, M. Kuhlen ${ }^{27}$, T. Kurča ${ }^{36}$, J. Kurzhöfer ${ }^{8}$, D. Lacour ${ }^{30}$, B. Laforge ${ }^{9}$, M.P.J. Landon ${ }^{21}$, W. Lange ${ }^{36}$, U. Langenegger ${ }^{37}$, A. Lebedev ${ }^{26}$, F. Lehner ${ }^{11}$, S. Levonian ${ }^{29}$, G. Lindström ${ }^{12}$, M. Lindstroem ${ }^{22}$, F. Linsel ${ }^{11}$, J. Lipinski ${ }^{13}$, B. List ${ }^{11}$, G. $\operatorname{Lobo}^{28}$, P. Loch ${ }^{11,43}$, J.W. Lomas ${ }^{23}$, G.C. Lopez ${ }^{12}$, V. Lubimov ${ }^{25}$, D. Lüke ${ }^{8,11}$, L. Lytkin ${ }^{14}$, N. Magnussen ${ }^{35}$, E. Malinovski ${ }^{26}$, R. Maraček ${ }^{18}$, P. Marage $^{4}$, J. Marks ${ }^{24}$, R. Marshall ${ }^{23}$, J. Martens ${ }^{35}$, G. Martin ${ }^{13}$, R. Martin ${ }^{20}$, H.-U. Martyn ${ }^{1}$, J. Martyniak ${ }^{6}$, T. Mavroidis ${ }^{21}$, S.J. Maxfield ${ }^{20}$, S.J. McMahon ${ }^{20}$, A. Mehta ${ }^{5}$, K. Meier ${ }^{16}$, F. Metlica ${ }^{14}$, A. Meyer $^{13}$, A. Meyer ${ }^{11}$, H. Meyer ${ }^{35}$, J. Meyer ${ }^{11}$, P.-O. Meyer ${ }^{2}$, A. Migliori29 ${ }^{29}$

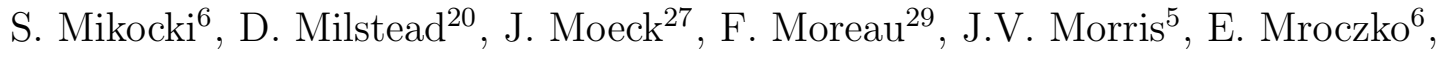


D. Müller ${ }^{38}$, G. Müller ${ }^{11}$, K. Müller ${ }^{11}$, P. Murín ${ }^{18}$, V. Nagovizin ${ }^{25}$, R. Nahnhauer ${ }^{36}$, B. Naroska ${ }^{13}$, Th. Naumann ${ }^{36}$, I. Négrii ${ }^{24}$, P.R. Newman ${ }^{3}$, D. Newton ${ }^{19}$, H.K. Nguyen ${ }^{30}$, T.C. Nicholls ${ }^{3}$, F. Niebergall ${ }^{13}$, C. Niebuhr ${ }^{11}$, Ch. Niedzballa ${ }^{1}$, H. Niggli ${ }^{37}$, G. Nowak ${ }^{6}$, G.W. Noyes ${ }^{5}$, T. Nunnemann ${ }^{14}$, M. Nyberg-Werther ${ }^{22}$, M. Oakden ${ }^{20}$, H. Oberlack ${ }^{27}$, J.E. Olsson ${ }^{11}$, D. Ozerov ${ }^{25}$, P. Palmen ${ }^{2}$, E. Panaro ${ }^{11}$, A. Panitch ${ }^{4}$, C. Pascaud ${ }^{28}$, G.D. Patel ${ }^{20}$, H. Pawletta ${ }^{2}$, E. Peppel ${ }^{36}$, E. Perez ${ }^{9}$, J.P. Phillips ${ }^{20}$, A. Pieuchot ${ }^{24}$, D. Pitzl ${ }^{37}$, G. Pope ${ }^{7}$, B. Povh ${ }^{14}$, S. Prell ${ }^{11}$, K. Rabbertz ${ }^{1}$, G. Rädel ${ }^{11}$, P. Reimer ${ }^{31}$, S. Reinshagen ${ }^{11}$, H. Rick ${ }^{8}$, F. Riepenhausen ${ }^{2}$, S. Riess ${ }^{13}$, E. Rizvi ${ }^{21}$, P. Robmann ${ }^{38}$, H.E. Roloff ${ }^{36, \dagger}$, R. Roosen ${ }^{4}$, K. Rosenbauer ${ }^{1}$, A. Rostovtsev ${ }^{25}$, F. Rouse ${ }^{7}$, C. Royon ${ }^{9}$, K. Rüter ${ }^{27}$, S. Rusakov ${ }^{26}$, K. Rybicki ${ }^{6}$, D.P.C. Sankey ${ }^{5}$, P. Schacht ${ }^{27}$, S. Schiek ${ }^{13}$, S. Schleif ${ }^{16}$, P. Schleper ${ }^{15}$, W. von Schlippe ${ }^{21}$, D. Schmidt ${ }^{35}$, G. Schmidt ${ }^{13}$, L. Schoeffel ${ }^{9}$, A. Schöning ${ }^{11}$, V. Schröder ${ }^{11}$, E. Schuhmann ${ }^{27}$, B. Schwab ${ }^{15}$, F. Sefkow ${ }^{38}$, R. Sell ${ }^{11}$, A. Semenov ${ }^{25}$, V. Shekelyan ${ }^{11}$, I. Sheviakov ${ }^{26}$, L.N. Shtarkov ${ }^{26}$, G. Siegmon ${ }^{17}$, U. Siewert ${ }^{17}$, Y. Sirois ${ }^{29}$, I.O. Skillicorn ${ }^{10}$, P. Smirnov ${ }^{26}$, V. Solochenko ${ }^{25}$, Y. Soloviev ${ }^{26}$, A. Specka ${ }^{29}$, J. Spiekermann ${ }^{8}$, S. Spielman ${ }^{29}$, H. Spitzer ${ }^{13}$, F. Squinabol ${ }^{28}$, P. Steffen ${ }^{11}$, R. Steinberg ${ }^{2}$, H. Steiner ${ }^{11,40}$, J. Steinhart ${ }^{13}$, B. Stella ${ }^{33}$, A. Stellberger ${ }^{16}$, J. Stier ${ }^{11}$,

J. Stiewe ${ }^{16}$, U. Stößlein ${ }^{36}$, K. Stolze ${ }^{36}$, U. Straumann ${ }^{15}$, W. Struczinski ${ }^{2}$, J.P. Sutton ${ }^{3}$, S. Tapprogge ${ }^{16}$, M. Taševský ${ }^{32}, \mathrm{~V}$. Tchernyshov ${ }^{25}, \mathrm{~S}$. Tchetchelnitski ${ }^{25}, \mathrm{~J}$. Theissen ${ }^{2}$, C. Thiebaux ${ }^{29}$, G. Thompson ${ }^{21}$, N. Tobien ${ }^{11}$, R. Todenhagen ${ }^{14}$, P. Truöl ${ }^{38}$, G. Tsipolitis ${ }^{37}$, J. Turnau ${ }^{6}$, J. Tutas ${ }^{15}$, E. Tzamariudaki ${ }^{11}$, P. Uelkes ${ }^{2}$, A. Usik ${ }^{26}$, S. Valkár ${ }^{32}$, A. Valkárová ${ }^{32}$, C. Vallée ${ }^{24}$, D. Vandenplas ${ }^{29}$, P. Van $\mathrm{Esch}^{4}$, P. Van Mechelen ${ }^{4}$, Y. Vazdik ${ }^{26}$, P. Verrecchia ${ }^{9}$, G. Villet ${ }^{9}$, K. Wacker ${ }^{8}$, A. Wagener ${ }^{2}$, M. Wagener ${ }^{34}$, B. Waugh ${ }^{23}$, G. Weber ${ }^{13}$, M. Weber ${ }^{16}$, D. Wegener ${ }^{8}$, A. Wegner ${ }^{27}$, T. Wengler ${ }^{15}$, M. Werner ${ }^{15}$, L.R. West ${ }^{3}$, T. Wilksen ${ }^{11}$, S. Willard ${ }^{7}$, M. Winde ${ }^{36}$, G.-G. Winter ${ }^{11}$, C. Wittek ${ }^{13}$, M. Wobisch ${ }^{2}$, E. Wünsch ${ }^{11}$, J. Žáček ${ }^{32}$, D. Zarbock ${ }^{12}$, Z. Zhang ${ }^{28}$, A. Zhokin ${ }^{25}$, P. Zini ${ }^{30}$, F. Zomer ${ }^{28}$, J. Zsembery ${ }^{9}$, K. Zuber ${ }^{16}$, and M. zurNedden ${ }^{38}$

${ }^{1}$ I. Physikalisches Institut der RWTH, Aachen, Germany ${ }^{a}$

${ }^{2}$ III. Physikalisches Institut der RWTH, Aachen, Germany ${ }^{a}$

${ }^{3}$ School of Physics and Space Research, University of Birmingham, Birmingham, UK ${ }^{b}$

${ }^{4}$ Inter-University Institute for High Energies ULB-VUB, Brussels; Universitaire

Instelling Antwerpen, Wilrijk; Belgium ${ }^{c}$

${ }^{5}$ Rutherford Appleton Laboratory, Chilton, Didcot, $U K^{b}$

${ }^{6}$ Institute for Nuclear Physics, Cracow, Poland ${ }^{d}$

${ }^{7}$ Physics Department and IIRPA, University of California, Davis, California, USA

${ }^{8}$ Institut für Physik, Universität Dortmund, Dortmund, Germany ${ }^{a}$

${ }^{9}$ CEA, DSM/DAPNIA, CE-Saclay, Gif-sur-Yvette, France

${ }^{10}$ Department of Physics and Astronomy, University of Glasgow, Glasgow, UK

${ }^{11}$ DESY, Hamburg, Germany ${ }^{a}$

12 I. Institut für Experimentalphysik, Universität Hamburg, Hamburg, Germany ${ }^{a}$

${ }^{13}$ II. Institut für Experimentalphysik, Universität Hamburg, Hamburg, Germany ${ }^{a}$

${ }^{14}$ Max-Planck-Institut für Kernphysik, Heidelberg, Germany ${ }^{a}$

${ }^{15}$ Physikalisches Institut, Universität Heidelberg, Heidelberg, Germany ${ }^{a}$

${ }^{16}$ Institut für Hochenergiephysik, Universität Heidelberg, Heidelberg, Germany ${ }^{a}$ 
17 Institut für Reine und Angewandte Kernphysik, Universität Kiel, Kiel, Germany ${ }^{a}$

18 Institute of Experimental Physics, Slovak Academy of Sciences, Košice, Slovak

Republic ${ }^{f, j}$

19 School of Physics and Chemistry, University of Lancaster, Lancaster, $U K^{b}$

20 Department of Physics, University of Liverpool, Liverpool, UK ${ }^{b}$

21 Queen Mary and Westfield College, London, UK ${ }^{b}$

22 Physics Department, University of Lund, Lund, Sweden ${ }^{g}$

23 Physics Department, University of Manchester, Manchester, UK ${ }^{b}$

${ }^{24}$ CPPM, Université d'Aix-Marseille II, IN2P3-CNRS, Marseille, France

25 Institute for Theoretical and Experimental Physics, Moscow, Russia

${ }^{26}$ Lebedev Physical Institute, Moscow, Russia ${ }^{f}$

27 Max-Planck-Institut für Physik, München, Germany ${ }^{a}$

${ }^{28}$ LAL, Université de Paris-Sud, IN2P3-CNRS, Orsay, France

${ }^{29}$ LPNHE, Ecole Polytechnique, IN2P3-CNRS, Palaiseau, France

30 LPNHE, Universités Paris VI and VII, IN2P3-CNRS, Paris, France

31 Institute of Physics, Czech Academy of Sciences, Praha, Czech Republic ${ }^{f, h}$

32 Nuclear Center, Charles University, Praha, Czech Republic ${ }^{f, h}$

33 INFN Roma 1 and Dipartimento di Fisica, Università Roma 3, Roma, Italy

34 Paul Scherrer Institut, Villigen, Switzerland

35 Fachbereich Physik, Bergische Universität Gesamthochschule Wuppertal, Wuppertal, Germany $^{a}$

${ }^{36}$ DESY, Institut für Hochenergiephysik, Zeuthen, Germany ${ }^{a}$

37 Institut für Teilchenphysik, ETH, Zürich, Switzerland

38 Physik-Institut der Universität Zürich, Zürich, Switzerland ${ }^{i}$

39 Visitor from Physicss Dept. University Louisville, USA

40 On leave from LBL, Berkeley, USA

${ }^{41}$ Institut für Physik, Humboldt-Universität, Berlin, Germany ${ }^{a}$

42 Rechenzentrum, Bergische Universität Gesamthochschule Wuppertal, Wuppertal, Germany ${ }^{a}$

43 Physics Department, University of Arizona, Tuscon, USA

$\dagger$ Deceased

a Supported by the Bundesministerium für Bildung, Wissenschaft, Forschung und Technologie, FRG, under contract numbers 6AC17P, 6AC47P, 6DO57I, 6HH17P, 6HH27I, 6HD17I, 6HD27I, 6KI17P, 6MP17I, and 6WT87P

${ }^{b}$ Supported by the UK Particle Physics and Astronomy Research Council, and formerly by the UK Science and Engineering Research Council

c Supported by FNRS-NFWO, IISN-IIKW

${ }^{d}$ Supported by the Polish State Committee for Scientific Research, grant nos. 115/E-743/SPUB/P03/109/95 and 2 P03B 244 08p01, and Stiftung für

Deutsch-Polnische Zusammenarbeit, project no. 506/92

e Supported in part by USDOE grant DE F603 91 ER 40674

${ }^{f}$ Supported by the Deutsche Forschungsgemeinschaft

$g$ Supported by the Swedish Natural Science Research Council

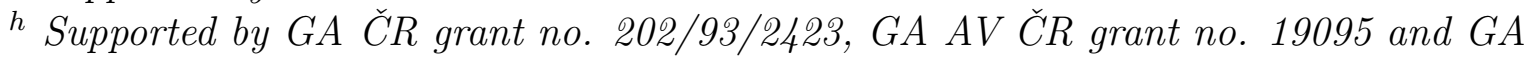


UK grant no. 342

${ }^{i}$ Supported by the Swiss National Science Foundation

$j$ Supported by VEGA SR grant no. 2/1325/96 


\section{Introduction}

The electron-proton collider HERA allows to explore a new kinematic region in deep inelastic scattering (DIS) down to the very small Bjorken- $x$ of about $10^{-5}$, where new dynamic features of QCD may show up. So far, the description of the nucleon structure function measurement by perturbative QCD, cast into the DGLAP (Dokshitzer-GribovLipatov-Altarelli-Parisi) parton evolution equations [1], has been extremely successful, and constitutes one of the major successes of QCD. At small enough $x$, however, these equations are expected to cease to be a good approximation. For the small $x$ regime the BFKL (Balitsky-Fadin-Kuraev-Lipatov) equation [2] has been suggested, and it would be very interesting to test QCD in such a new regime. To lowest order the BFKL and DGLAP equations resum the leading logarithmic $\left(\alpha_{s} \ln (1 / x)\right)^{n}$ and $\left(\alpha_{s} \ln \left(Q^{2} / Q_{0}^{2}\right)\right)^{n}$ contributions respectively, where $Q^{2}$ is the invariant mass squared of the virtual photon and $Q_{0}$ is the cut-off for the perturbative evolution. In these approximations the leading diagrams are of the ladder type (Fig. 1). The leading log DGLAP resummation corresponds to a strong ordering of the transverse momenta $k_{T}$ (w.r.t. the proton beam) in the parton cascade $\left(Q_{0}^{2} \ll k_{T 1}^{2} \ll \ldots k_{T i}^{2} \ll \ldots Q^{2}\right)$. In the BFKL regime, the transverse momenta follow a kind of random walk $\left(k_{T i}^{2} \approx k_{T i+1}^{2}\right)[3]$.

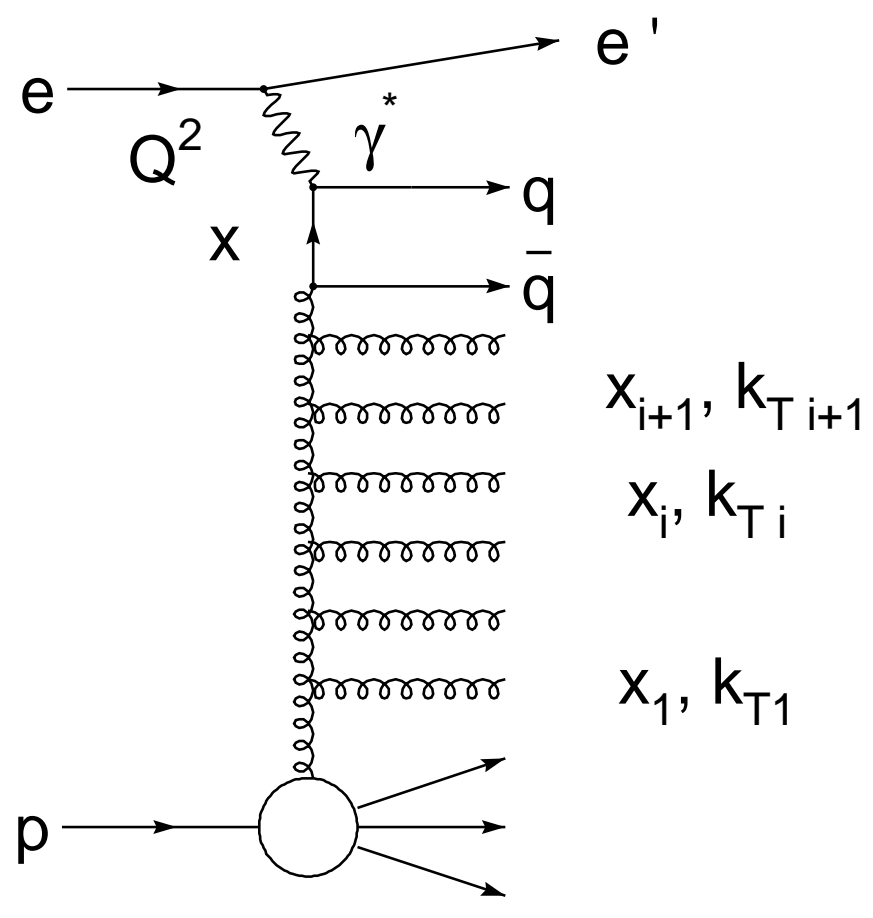

Figure 1: Generic diagram for parton evolution.

The strong rise towards small $x$ of the structure function $F_{2}$ measured at HERA [4, 5] can be described with DGLAP evolution [6, 7] and is also consistent with the BFKL expectation. Less inclusive measurements, namely of the hadronic final state emerging from the cascade, may offer more sensitive means of searching for BFKL evolution. One 
possible signature is the quantity of transverse energy, $E_{T}$, produced in a region between the current (struck) quark and the proton remnant [8]. As a consequence of the strong $k_{T}$ ordering, DGLAP evolution is expected to produce less $E_{T}$ than BFKL evolution [9]. The HERA $E_{T}$ flow data [10] can be interpreted consistently within the BFKL framework, but from these data alone it is not possible to disentangle non-perturbative hadronization effects from perturbative parton radiation, precluding an unambiguous test of the underlying parton dynamics [11, 12. In fact, the data can also be described with a DGLAP based parton shower model with the assumption that the hadronization effects are large [12].

In this paper complementary measurements of charged particle transverse momentum $\left(p_{T}\right)$ spectra are presented. Such spectra have been proposed 13 as a more direct probe of the underlying parton dynamics than the $E_{T}$ flow measurements. It has been shown with the aid of QCD models that the high- $p_{T}$ tail is sensitive to parton radiation, whereas the production of hard particles from hadronization is suppressed. For the $k_{T}$ unordered scenario a harder $p_{T}$ spectrum is expected in the central region than for the $k_{T}$ ordered evolution, because parton emissions are less restricted in $k_{T}$. The unsuppressed gluon radiation should give rise to a hard tail of the $p_{T}$ distribution for the hadrons emerging from the cascade. The tail would be absent if the $E_{T}$ seen in the data stems predominantly from hadronization. A particular good discrimination can be obtained with events in which $E_{T}$ is large. Radiation of a hard gluon may lead to large $E_{T}$ and a high $p_{T}$ particle, but if hadronization were responsible for the large $E_{T}$, many soft particles are expected instead. Furthermore, a different $x$-behaviour of the $p_{T}$ spectra is predicted. The phase space increases with $W^{2} \approx Q^{2} / x$ ( $W$ is the invariant mass of the hadronic final state) and therefore one may expect more gluon radiation, more $E_{T}$ and harder $p_{T}$ spectra with decreasing $x$, but not if $k_{T}$ ordering or another mechanism is invoked to suppress gluon radiation. The measurements presented here are compared to predictions from Monte Carlo models with different mechanisms for gluon radiation. They incorporate QCD evolution in different approximations and utilize phenomenological models for the non-perturbative hadronization phase.

The MEPS model (Matrix Element plus Parton Shower) incorporated within the LEPTO generator [14, uses the first order QCD matrix elements, with additional soft emissions generated by adding leading log DGLAP parton showers. Though this approach is widely used with considerable success, it has to be pointed out that the DGLAP formalism was derived for the totally inclusive structure function evolution, and it can be questioned whether it is fully applicable for exclusive final states [12]. In the colour dipole model (CDM) [15], as implemented in ARIADNE [16], radiation stems from colour dipoles formed by the colour charges. Both programs use the Lund string model [17] for hadronizing the partonic final state. The HERWIG model [18 is also based on leading log parton showers, with additional matrix element corrections [19]. This model implements an alternative (cluster) fragmentation scheme [20]. The CDM description of gluon emission is similar to that of BFKL evolution to the extent that the gluons emitted by the dipoles do not obey strong ordering in $k_{T}$ [21]. In the MEPS and HERWIG models the partons emitted in the cascade are strongly ordered in $k_{T}$, because they are based on leading log DGLAP parton showers. The models are used in their versions LEPTO 6.4 for MEPS, ARIADNE 4.08 for CDM and HERWIG 5.8, together with the parton density parameterization GRV [7]. They provide a satisfactory overall description of current DIS 
final state data [22], in particular of the $E_{T}$ flows f. For this analysis the use of the alternative parameterization MRS-H [24] would give model predictions which differ only slightly from those shown.

\section{Detector Description}

A detailed description of the H1 apparatus can be found elsewhere [25]. The following briefly describes the detector components relevant to this analysis.

The hadronic energy flow and the scattered positron are measured with a liquid argon (LAr) calorimeter and a backward electromagnetic lead-scintillator calorimeter (BEMC) respectively. The LAr calorimeter [26] extends over the polar angular range $4^{\circ}<\theta<153^{\circ}$ with full azimuthal coverage, where $\theta$ is defined with respect to the proton beam direction $(+z$ axis). The depth varies between 4.5 and 8 interaction lengths for $\theta<125^{\circ}$. Test beam measurements of the LAr calorimeter modules show an energy resolution of $\sigma_{E} / E \approx 0.50 / \sqrt{E[\mathrm{GeV}]} \oplus 0.02$ for charged pions [27]. The absolute scale of the hadronic energy measurement is presently known to $5 \%$, as determined from studies of the transverse momentum $\left(p_{T}\right)$ balance in DIS events.

The BEMC [28 (with a depth of 22.5 radiation lengths and 1 interaction length) covers the backward region, $151^{\circ}<\theta<177^{\circ}$. A major task of the BEMC is to trigger and to measure precisely scattered positrons in DIS processes with $Q^{2}$ values ranging from 5 to $100 \mathrm{GeV}^{2}$. The BEMC energy scale for electrons is known to an accuracy of $1 \%$. Its resolution is given by $\sigma_{E} / E=0.10 / \sqrt{E[\mathrm{GeV}]} \oplus 0.42 / E[\mathrm{GeV}] \oplus 0.03$ [28. A backward proportional chamber (BPC), in front of the BEMC, with an angular acceptance of $155.5^{\circ}<\theta<174.5^{\circ}$, serves to identify electrons and to measure precisely their direction. Using information from the $\mathrm{BPC}$, the $\mathrm{BEMC}$ and the reconstructed event vertex, the polar angle of the scattered positron is known to a precision of $1 \mathrm{mrad}$.

The calorimeters are surrounded by a superconducting solenoid which provides a uniform magnetic field of $1.15 \mathrm{~T}$ parallel to the beam axis in the tracking region.

The tracking system consists of a central and a forward part. The central tracking system is mounted concentrically around the beam line, covering polar angles of $20^{\circ}<\theta<$ $160^{\circ}$. Charges and momenta of charged particles are measured by two coaxial cylindrical drift chambers (central jet chamber, CJC), providing up to 56 space points in the radial plane. Longitudinal coordinates are obtained via charge division from the CJC, and from dedicated drift chambers which are interleaved with the CJC. For the CJC the resolutions achieved are $\sigma_{p_{T}} / p_{T} \approx 0.009 \cdot p_{T}[\mathrm{GeV}] \oplus 0.015$ and $\sigma_{\theta}=20 \mathrm{mrad}$ [25]. The central tracking system is complemented by the forward tracker, which is built in three sections covering a polar angle range of $7^{\circ}<\theta<25^{\circ}$. Each section is made up of a series of multiwire proportional and drift chambers which are arranged to facilitate track reconstruction at small angles to the proton beam. For tracks fitted to a vertex the resolution has been

${ }^{1}$ However, in LEPTO (among other things) the new concept of soft colour interactions [12] had to be introduced to reproduce the level of $E_{T}$ seen in the data [8, 22, 12]. Intriguingly, this mechanism also produces rapidity gap events [23] at a rate of about 10\%, comparable to observation [12]. Also the cluster fragmentation scheme in HERWIG produces rapidity gap events. In this paper rapidity gap events are excluded. 
shown to be $\sigma_{p_{T}} / p_{T} \approx 0.02 \cdot p_{T}[\mathrm{GeV}] / \sin \theta \oplus 0.1$ and the angular resolution, $\sigma_{\theta}$, to be better than $1 \mathrm{mrad}$ [29].

Scintillation counters installed behind the BEMC are used to reject proton induced background. Small angle electron/photon taggers are used for luminosity measurements and for the study of photoproduction background.

\section{$3 \quad$ Event and Track Selection}

The data used in this analysis were collected in 1994, with positrons of energy $E_{e}=$ $27.5 \mathrm{GeV}$ colliding with protons of energy $E_{p}=820 \mathrm{GeV}$, resulting in a total centre of mass energy of $\sqrt{s}=300 \mathrm{GeV}$. The data correspond to an integrated luminosity of $1.3 \mathrm{pb}^{-1}$. For this analysis DIS events with $5<Q^{2}<50 \mathrm{GeV}^{2}$ are used, in which the scattered positron is observed in the BEMC. The events are triggered by requiring a cluster of more than $4 \mathrm{GeV}$ in the BEMC. After reconstruction, DIS events are selected in the following way:

- The scattered positron, defined as the most energetic BEMC cluster, must have an energy $E_{e}^{\prime}$ larger than $12 \mathrm{GeV}$ and a polar angle $\theta_{e}$ below $173^{\circ}$ in order to ensure high trigger efficiency and a small photoproduction background [ 4 .

- The lateral size of the positron cluster, calculated as the energy weighted radial distance of the cells from the cluster centre, has to be smaller than $4 \mathrm{~cm}$. The cluster must be associated with a reconstructed BPC space point Further reduction of photoproduction background and the removal of events in which an energetic photon is radiated off the incoming positron is achieved by requiring $\sum_{j}\left(E_{j}-p_{z, j}\right)>$ $30 \mathrm{GeV}$ [四], with the sum extending over all particles $j$ (measured calorimetrically) in the event.

- The radial coordinate of the $\mathrm{BPC}$ hit must be less than $60 \mathrm{~cm}$, corresponding to a positron angle above $157^{\circ}$ with respect to the nominal interaction point, ensuring full containment of the positron shower in the BEMC.

- The $z$ position of the event vertex reconstructed from charged tracks has to be within $30 \mathrm{~cm}$ of the average of all collision events.

- The energy in the forward region $\left(4.4^{\circ}<\theta<15^{\circ}\right)$ has to be larger than $0.5 \mathrm{GeV}$ in order to exclude diffractive events with large rapidity gaps in the forward region [23, 8].

- Remaining background is rejected by requiring no veto from the time-of-flight counters.

The kinematic variables are determined using information from the scattered positron: $Q^{2}=4 E_{e} E_{e}^{\prime} \cos ^{2}\left(\theta_{e} / 2\right)$ and $y=1-\left(E_{e}^{\prime} / E_{e}\right) \cdot \sin ^{2}\left(\theta_{e} / 2\right)$. The scaling variable $x$ is then derived via $x=Q^{2} /(y s)$, and the hadronic invariant mass squared is $W^{2}=s y-Q^{2}$. 


\begin{tabular}{|c|c|c|c|c|c|c|}
\hline Kin.bin & $x / 10^{-3}$ & $Q^{2} / \mathrm{GeV}^{2}$ & $\langle x\rangle / 10^{-3}$ & $\left\langle Q^{2}\right\rangle / \mathrm{GeV}^{2}$ & $\left\langle W^{2}\right\rangle / \mathrm{GeV}^{2}$ & \#events \\
\hline & & & & & & \\
0 & $0.1-10$ & $5-50$ & 1.14 & 18.3 & 24975 & 59463 \\
\hline \hline 1 & $0.1-0.2$ & $5-10$ & 0.16 & 7.0 & 45296 & 4853 \\
2 & $0.2-0.5$ & $6-10$ & 0.29 & 8.8 & 31686 & 6294 \\
\hline 3 & $0.2-0.5$ & $10-20$ & 0.37 & 13.1 & 36893 & 9564 \\
4 & $0.5-0.8$ & $10-20$ & 0.64 & 14.0 & 22401 & 7129 \\
5 & $0.8-1.5$ & $10-20$ & 1.1 & 14.3 & 13498 & 7964 \\
6 & $1.5-4.0$ & $10-20$ & 2.1 & 15.3 & 7543 & 4720 \\
\hline 7 & $0.5-1.4$ & $20-50$ & 0.93 & 28.6 & 32390 & 8349 \\
8 & $1.4-3.0$ & $20-50$ & 2.1 & 31.6 & 16025 & 6752 \\
9 & $3.0-10$ & $20-50$ & 4.4 & 34.7 & 8225 & 3838 \\
\hline
\end{tabular}

Table 1: The kinematic intervals and the average $x, Q^{2}$ and $W^{2}$ from the raw data. The numbers of events surviving the selections are given.

- As the precision of the $y$ measurement degrades with $1 / y$, a cut $y>0.05$ is imposed. Events in which the positron is poorly reconstructed, or in which an energetic photon has been radiated from the incoming positron, are removed by demanding that the value of $y$ determined using the hadrons $y_{h}=\sum\left(E-p_{z}\right) / 2 E_{e}$, where the sum runs over all hadronic energy deposits, also be greater than 0.05 .

Charged particle tracks are measured in the forward tracker and the CJC. They are required to originate from the primary vertex and their polar angle must lie between $8^{\circ}$ and $155^{\circ}$. The upper limit ensures that the scattered positron does not enter the sample of charged hadronic tracks. Central tracks are required to have a radial track length of at least $10 \mathrm{~cm}$ to ensure good momentum resolution and a measured point in the inner CJC. Forward tracks are required to be well reconstructed and to have an acceptable link $\chi^{2}$ in the overlap region between the forward and central trackers. After having selected such high quality tracks, the efficiency for finding a genuine primary track with $p_{T}^{\text {lab }}>0.15$ $\mathrm{GeV}$ is better than $93 \%$ for central tracks and $70 \%$ for forward tracks.

\section{Results}

The results are presented for the hadronic centre of mass system (CMS), i.e. the rest system of the proton and the exchanged boson, where the direction of the exchanged boson defines the positive $z^{\prime}$ axis. The event sample is divided into 9 different kinematic bins, resulting in three slices with almost constant $Q^{2}$ and varying $x$ (see Table (1). The same binning is used for the measurement of transverse energy flows [10].

The data are corrected bin-by-bin for detector effects, including photon conversions, secondary interactions, geometrical acceptance and for decay products of particles with a lifetime greater than $8 \mathrm{~ns}$ (e.g. $K_{s}^{0} \rightarrow \pi^{+} \pi^{-}$and $\Lambda \rightarrow p \pi$ ), as well as for QED radiative 
effects. The corrections are determined using Monte Carlo generated events f with a full simulation of the $\mathrm{H} 1$ detector response. The detector simulation has been checked by comparing in great detail distributions of track quality properties to the data. A visual scan of tracks in real and in simulated events leads to the conclusion that the tracker efficiency is simulated to an accuracy of better than $2 \%$ for central tracks and $10 \%$ for forward tracks. No spurious tracks due to noise hits have been found. No significant difference was found between the $p_{T}$ spectra of positively and negatively charged tracks.

Transverse momentum spectra are measured with the central tracker for the pseudorapidity interval $1.5<\eta<2.5$, where $\eta=-\ln \tan \left(\theta^{*} / 2\right)$, and $\theta^{*}$ is the angle with respect to the virtual photon direction. The lower $\eta$ limit is determined by the requirement of good acceptance in all 9 kinematic bins, and the upper limit restricts the measurement to a region away from the current fragmentation region. That is where significant differences between the different QCD evolution scenarios are expected.

The measured spectra, steeply falling with $p_{T}$, are shown in Fig. 2 for the nine kinematic bins and for the combined event sample. All distributions shown in this paper are normalized to the number of events $N$ that enter the distribution. The inner error bar represents the statistical error and the outer error bar the quadratic sum of statistical and systematic errors (except for Fig. 3, where only the total error is shown). We first discuss the systematic errors and then turn to a discussion of the results.

The correction factors applied to the original distributions are never larger than 2 or smaller than 0.5 and in most cases they are between 0.9 and 1.1 . The $p_{T}$ bins are much larger than the experimental resolution and bin centre corrections are applied. The boost to the CMS introduces a well understood change of the $p_{T}$ of the tracks of less than $10 \%$ from the laboratory value. The largest corrections are those necessary to compensate for event migration effects (contributing mainly at large $p_{T}$ ) and track selection cuts and detector acceptance (contributing mainly at small $p_{T}$ ). The main sources of systematic uncertainty are:

- The model dependence of the acceptance corrections (ARIADNE versus LEPTO). These differ by as much as $20 \%$, but in general agree to within $5 \%$.

- The track selection cuts. Variation of these leads to a $10 \%$ systematic error for particles with $p_{T}<0.5 \mathrm{GeV}$ and for the kinematic bins at low $W$, where measurements are close to the limit of the detector acceptance.

- A contamination of up to $5 \%$ from photoproduction for the kinematic bins at large $W$ [33]. In order to estimate the uncertainty due to this contamination, stricter electron quality cuts have been applied at the cost of statistics, and events with signals in the electron/photon taggers are rejected. Differences in the result obtained with

2 The event generator used is DJANGO [30] which simulates electroweak interactions using the HERACLES 31] algorithm and QCD corrections using the ARIADNE [16] program. The detector response is simulated using a program based on the GEANT [32] package. The Monte Carlo events are reconstructed in the same manner as the $e^{+} p$ collision data. The simulated and reconstructed events obtained using DJANGO describe the raw data spectra very well. Differences in the correction factors obtained with the alternative model LEPTO, which describes the data less well, are reflected in the systematic errors. 
respect to the standard selection are reflected in the systematic error and are up to $30 \%$ in kinematic bin 1, below $10 \%$ in bin 2 and below $5 \%$ in the other bins.

- The uncertainty of the BEMC energy scale. This affects the boost to the CMS. However, the effect is negligible for all bins except for those at large $x$ and $Q^{2}$, where it may give rise to an uncertainty up to $15 \%$.

The $p_{T}$ spectra shown in Fig. 2 are compared to the models ARIADNE, LEPTO and HERWIG, representing the suppressed (LEPTO, HERWIG) and unsuppressed (ARI$\mathrm{ADNE}$ ) gluon radiation scenarios. At "large" $x$ and $Q^{2}$ (here "large" means $Q^{2} \approx 35 \mathrm{GeV}^{2}$ and $x \approx 0.004$ ) all models provide a good description of the data. At smaller $x$ and $Q^{2}$, LEPTO and HERWIG fall significantly below the data for $p_{T}>1 \mathrm{GeV}$. ARIADNE gives a good description of the data over the full kinematic range. As shown in [13], the hardness of the $p_{T}$ spectrum can be related to the parton radiation. The shortfall of the models with suppressed gluon radiation indicates that at small $x$ there is more high $k_{T}$ parton radiation present than is produced by the models based upon leading log DGLAP parton showers. The data are well described by the ARIADNE model in which parton radiation is more abundant.

In order to look for the predicted hardening of the $p_{T}$ spectrum with decreasing $x$ due to increasing gluonic activity, the $p_{T}$ spectra are compared in Fig. 3a for the highest and lowest $x$ bins (differing by a factor 6 in $x$ ) of the intermediate $Q^{2}\left(\approx 14 \mathrm{GeV}^{2}\right)$ slice. There is a trend consistent with the prediction of the ARIADNE model, however not very significant. Because $W^{2} \approx Q^{2} / x$, this effect can also be understood as a $W$ dependence.

From a comparison of bins 2 and 7 (Fig. $3 \mathrm{~b}$ ), where $W^{2}$ is fixed $\left(\approx 32000 \mathrm{GeV}^{2}\right)$ and $Q^{2}$ varies by a factor 3 (from 9 to $29 \mathrm{GeV}^{2}$ ), it can be seen that the $p_{T}$ spectrum becomes harder with increasing $Q^{2}$. When $x$ is fixed $(x=0.0021)$, and $Q^{2}$ increased by a factor 2 , the $p_{T}$ spectrum also becomes harder, see Fig. 3 $\mathrm{c}$. We have observed previously that in the current fragmentation region the transverse energy, $E_{T}$, increases with $Q^{2}$ for fixed $W$ [34] and that the $Q^{2}$ dependence diminishes towards the proton remnant direction. The $E_{T}$ measured in the interval $1.5<\eta<2.5$ still increases slightly with $Q^{2}$. In the central interval $-0.5<\eta<0.5$ no $Q^{2}$ dependence of $E_{T}$ is seen for $Q^{2}<50 \mathrm{GeV}^{2}$. To summarize, at fixed $Q^{2}$ there appears to be a hardening of the $p_{T}$ spectrum with decreasing $x$ and correspondingly increasing $W$, while at fixed $W$ there is a hardening for increasing $Q^{2}$.

Both the forward tracker and the CJC are used to measure the $p_{T}$ spectrum in the more central region $0.5<\eta<1.5$ (see Fig. 4), where the difference between the two types of models becomes stronger. Bins 6 and 9 contain roughly equal numbers of forward and central tracks whereas the remaining bins are based on central tracks alone since they contain few forward tracks. In addition to the sytematic errors described above, the data in bins 6 and 9 have a contribution from a 10\% uncertainty in the forward tracker reconstruction efficiency. The failure of the event generators which are based on leading log DGLAP parton showers to describe the hard component present in the data now becomes more apparent. ARIADNE describes the data well. Comparing the $p_{T}$ spectra for the two pseudorapidity intervals in the same kinematic bin (bin 3, low $x$ ), it is found that the spectrum becomes harder when one moves towards the current direction, see Fig. 3d. This is not surprising, as the average $k_{T}$ of the gluons emitted in both scenarios, DGLAP and BFKL, increases along the ladder towards the photon vertex [3]. Also, 
radiation associated with the current system, for which the relevant scale is the virtuality of the photon $\left(Q^{2}\right)$, is expected to contribute more in that direction.

For the LEPTO predictions in this paper we modified some LEPTO parameters to improve somewhat the description of the data over the standard setting. The divergency cut-off parameters for the matrix element were changed s and a fragmentation parameter set tuned to the LEP data [36] was used. The most sensitive parameter, however, is the virtuality cut-off below which final state radiation is not allowed, and fragmentation takes over. If this parameter would be changed from its default value of $1 \mathrm{GeV}^{2}$ to 3 $\mathrm{GeV}^{2}$, a much better description of the $p_{T}$ spectra for $1.5<\eta<2.5$ could be achieved. However, this setting would result in a poor description of the observed scaling violations in the Feynman- $x$ spectra [8, 37]. In addition, the $p_{T}$ spectra measured more centrally at $0.5<\eta<1.5$ are still much harder than in the modified model.

In order to obtain a measure for the hardness of the $p_{T}$ spectrum over the entire range of pseudorapidity accessible to this analysis, the average multiplicity of charged particles with $p_{T}>1 \mathrm{GeV}$ is measured (see Fig. 5) as a function of pseudorapidity. The surplus of hard particles in the data over the DGLAP-like models at small $x$ and away from the current region is obvious. The best description of the data is achieved by ARIADNE. In contrast to the multiplicity of hard particles, the overall multiplicity, which is dominated by soft particles, is much better (although not perfectly) described by all models, see Fig. 6. Only the HERWIG model overshoots the data considerably at small $x$ and towards the central region.

The discrimination between the different scenarios can be enhanced by selecting events with substantial hadronic activity because, in such events, there is also substantial partonic activity for the model with unsuppressed radiation, as opposed to the other models. Here the transverse energy measured with the calorimeter in the $\eta$ range from 0.0 to 2.0 is required to be larger than $6 \mathrm{GeV}$. The $p_{T}$ distribution of the track with the largest transverse momentum in the range $0.5<\eta<1.5, p_{T}^{\max }$, in each event is then determined, see Fig. 7. In this way one can reveal the correlation between large $E_{T}$ production, which could be produced by many soft particles in the hadronization, and high $p_{T}$ particles, which indicate hard parton radiation. The events contain much harder particles than are produced in the models MEPS and HERWIG. Again, ARIADNE with enhanced parton radiation describes the data very well. The discrepancy between the data and ARIADNE on the one hand, and LEPTO and HERWIG on the other, becomes larger for smaller $x$ and $Q^{2}$. It is noteworthy that the colour dipole model (ARIADNE) is able to describe kinematic regions at both large and small $x$ simultaneously.

\section{Conclusions}

Transverse momentum spectra of charged particles in deep inelastic scattering are measured as a function of $x$ and $Q^{2}$ in the current and the central fragmentation regions. The average charged particle multiplicity is also measured as a function of pseudorapidity. The data are compared to QCD models incorporating different schemes for parton evolution in the proton, namely both with and without transverse momentum ordering of the parton

\footnotetext{
${ }^{3} \operatorname{parl}(8)$ changed from 0.01 to 0.04 and $\operatorname{parl}(9)$ from 1 to 4 35.
} 
emissions. The latter yields a larger amount of high $k_{T}$ parton radiation between the current and remnant systems. In this respect the results obtained using the models studied can be considered as representative of the expectations for the hadronic final state of the DGLAP and BFKL evolution schemes, respectively. At large $x, x>0.001$, all models provide an overall satisfactory description of the measured $p_{T}$ spectra. At small $x$ however and at central rapidity the $p_{T}$ distributions are significantly harder than expected from the models with suppressed radiation. The unsuppressed scenario, as realized in a colour dipole radiation model, is able to describe the data. The available models for the description of the hadronic final state in deep inelastic scattering based on the conventional leading log DGLAP equations are not able to describe the data. The observed hard $p_{T}$ spectra indicate hard parton radiation, which could be provided by BFKL-type contributions. Further interpretation of the data has to await firm QCD calculations and a Monte Carlo model which explicitly includes the BFKL terms.

Acknowledgements. We are grateful to the HERA machine group whose outstanding efforts made this experiment possible. We appreciate the immense effort of the engineers and technicians who constructed and maintained the detector. We thank the funding agencies for financial support. We acknowledge the support of the DESY technical staff. We also wish to thank the DESY directorate for the hospitality extended to the non-DESY members of the collaboration. 


\section{References}

[1] Yu. L. Dokshitzer, Sov. Phys. JETP 46 (1977) 641;

V.N. Gribov and L.N. Lipatov, Sov. J. Nucl. Phys. 15 (1972) 438 and 675;

G. Altarelli and G. Parisi, Nucl. Phys. 126 (1977) 297.

[2] E.A. Kuraev, L.N. Lipatov and V.S. Fadin, Sov. Phys. JETP 45 (1972) 199;

Y.Y. Balitsky and L.N. Lipatov, Sov. J. Nucl. Phys. 28 (1978) 282.

[3] J. Bartels, H. Lotter, Phys. Lett. B309 (1993) 400;

A. Mueller, Columbia preprint CU-TP-658 (1994);

J. Bartels, H. Lotter and M. Vogt, Phys. Lett. B373 (1996) 215.

[4] H1 Collab., T. Ahmed et al., Nucl. Phys. B439 (1995) 471.

[5] ZEUS Collab., M. Derrick et al., Z. Phys. C65 (1995) 379; Z. Phys. C69 (1996) 607.

[6] A.J. Askew, J. Kwieciński, A.D. Martin, P.J. Sutton, Phys. Lett. B325 (1994) 21.

[7] M. Glück, E. Reya, A. Vogt, Z. Phys. C67 (1995) 433.

[8] H1 Collab., I. Abt et al., Z. Phys. C63 (1994) 377.

[9] J. Kwieciński, A.D. Martin, P.J. Sutton and K.Golec-Biernat, Phys. Rev. D50 (1994) 217

K.Golec-Biernat, J. Kwieciński, A.D. Martin and P.J. Sutton, Phys. Lett. B335 (1994) 220.

[10] H1 Collab., S. Aid et al., Phys. Lett. B356 (1995) 118.

[11] M. Kuhlen, MPI-PhE/95-19 (1995), hep-ex/9508014; Proc. of the Workshop on Deep Inelastic Scattering and QCD - DIS95, Paris, April 1995, eds. JF. Laporte and Y. Sirois, p. 345.

[12] A. Edin, G. Ingelman and J. Rathsman, Phys.Lett. B366 (1996) 371;

A. Edin, G. Ingelman and J. Rathsman, DESY 96-060.

[13] M. Kuhlen, Phys. Lett. B382 (1996) 441;

M. Kuhlen, contribution to appear in the Proc. of the Workshop on "Future Physics at HERA", 1995-1996, eds. A. De Roeck, G. Ingelman and R. Klanner.

[14] G. Ingelman, Proc. of the Workshop on Physics at HERA, Hamburg 1991, eds. W. Buchmüller and G. Ingelman, vol. 3, p. 1366.

[15] G. Gustafson, Ulf Petterson, Nucl. Phys. B306 (1988);

G. Gustafson, Phys. Lett. B175 (1986) 453;

B. Andersson, G. Gustafson, L. Lönnblad, Ulf Petterson, Z. Phys. C43 (1989) 625.

[16] L. Lönnblad, Comp. Phys. Comm. 71 (1992) 15. 
[17] T. Sjöstrand, Comp. Phys. Comm. 39 (1986) 347;

T. Sjöstrand and M. Bengtsson, Comp. Phys. Comm. 43 (1987) 367;

T. Sjöstrand, CERN-TH-6488-92 (1992).

[18] G. Marchesini et al., Comp. Phys. Comm. 67 (1992) 465.

[19] M. Seymour, Lund preprint LU-TP-94-12 (1994);

M. Seymour, Nucl. Phys. B436 (1995) 443.

[20] B.R. Webber, Nucl. Phys. B238 (1984) 492.

[21] L. Lönnblad, Z. Phys. C65 (1995) 285;

A. H. Mueller, Nucl. Phys. B415 (1994) 373;

L. Lönnblad, CERN-TH/95-95.

[22] T. Carli, Proc. of the DIS96 Workshop, Rome 1996, ed. A. Nigro.

[23] ZEUS Collab., M. Derrick et al., Phys. Lett. B315 (1993) 481;

H1 Collab., T. Ahmed et al., Nucl.Phys. B429 (1994) 477.

[24] A.D. Martin, W.J. Stirling and R.G. Roberts, Proc. of the Workshop on Quantum Field Theory and Theoretical Aspects of High Energy Physics, eds. B. Geyer and E.M. Ilgenfritz (1993) p. 11.

[25] H1 Collab., I. Abt et al., DESY H1-96-01, accepted by Nucl. Instr. and Meth..

[26] H1 Calorimeter Group, B. Andrieu et al., Nucl. Instr. and Meth. A336 (1993) 460.

[27] H1 Calorimeter Group, B. Andrieu et al., Nucl. Instr. and Meth. A336 (1993) 499.

[28] H1 BEMC Group, J. Bán et al., Nucl. Instr. and Meth. A372 (1996) 399.

[29] H1 Forward Tracker Group, S. Burke et al., Nucl. Instr. and Meth. A373 (1996) 227.

[30] K. Charchula, G. Schuler, H. Spiesberger, Comp. Phys. Comm. 81 (1994) 381.

[31] A. Kwiatkowski, H. Spiesberger and H.-J. Möhring, Comp. Phys. Comm. 69 (1992) 155.

[32] R. Brun et al., GEANT3 User's Guide, CERN-DD/EE 84-1, Geneva (1987).

[33] H1 Collaboration, S. Aid et al., Nucl. Phys. B470 (1996) 3.

[34] H1 Collab., S. Aid et al., Phys. Lett. B358 (1995) 412.

[35] T. Sjöstrand, Comp. Phys. Comm. 39 (1986) 347;

T. Sjöstrand and M. Bengtsson, Comp. Phys. Comm. 43 (1987) 367, and for JETSET 7.3, T. Sjöstrand, CERN-TH-6488-92 (1992).

[36] I.G. Knowles et al., hep-ph/9601212.

[37] ZEUS Collab., M. Derrick et al., Z. Phys. C70 (1996) 1. 


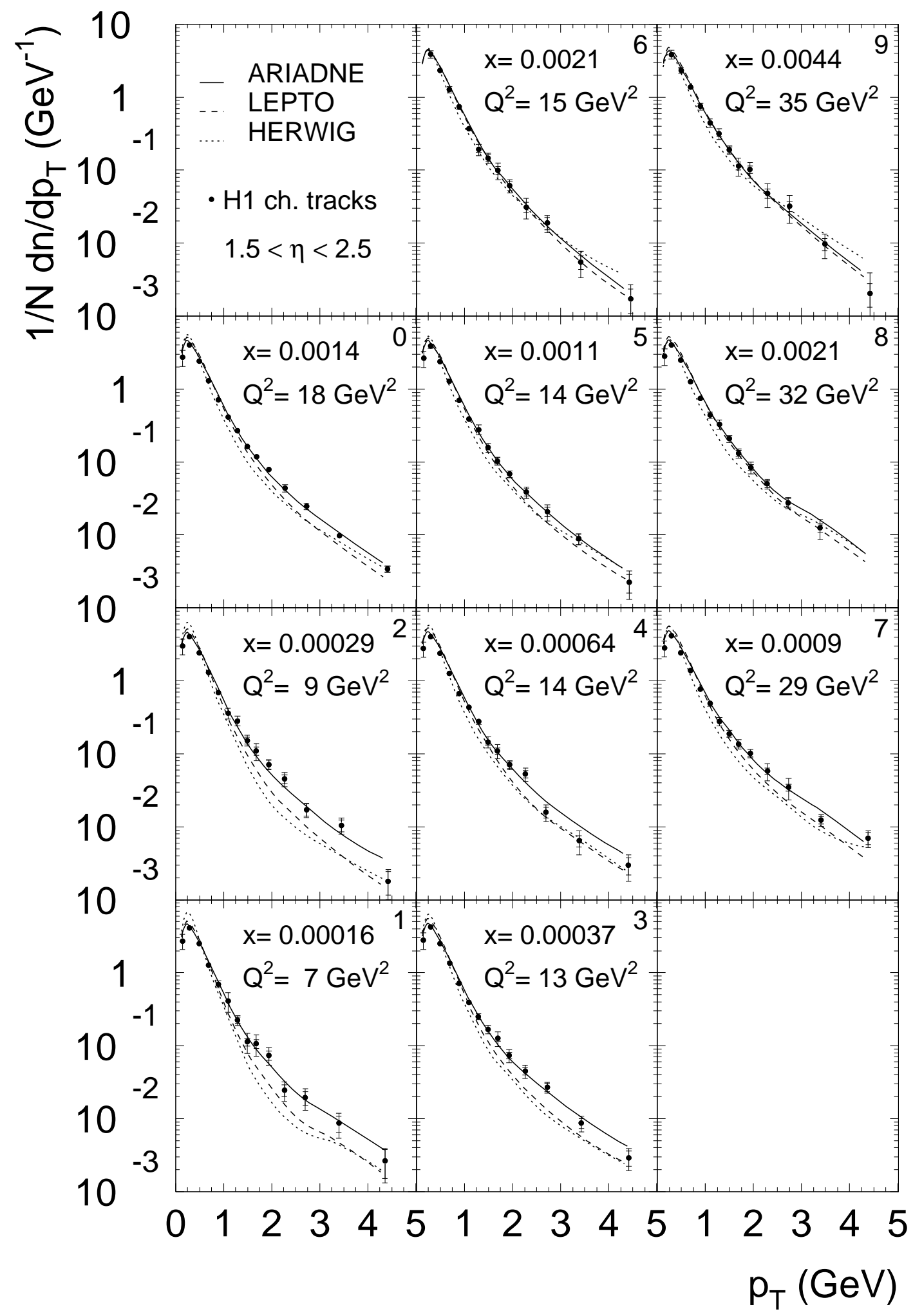

Figure 2: The transverse momentum spectra of charged particles, measured in the CMS in the pseudorapidity interval $1.5<\eta<2.5$. Data are shown for nine different kinematic bins (see Table 1) and the combined sample (bin 0). For comparison, the models ARIADNE (full line), LEPTO (dashed) and HERWIG (dotted) are overlayed. The mean values of $x$ and $Q^{2}$ are indicated. The inner error bars represent the statistical errors, the outer error bars the quadratic sum of statistical and systematic errors. 

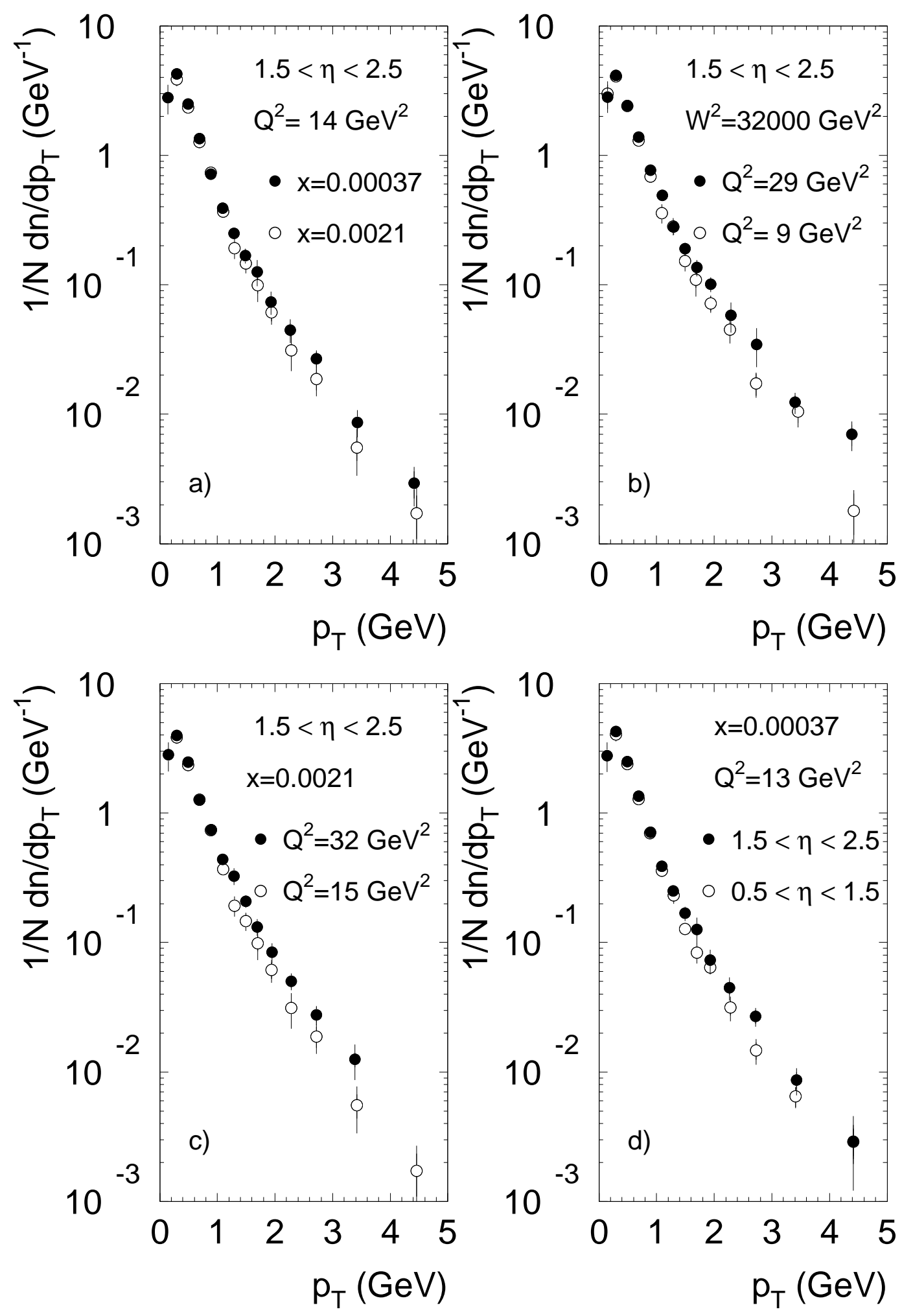

Figure 3: a) Comparison of the $p_{T}$ spectra at high $x$ (bin 6) and at low $x$ (bin 3) for fixed (intermediate) $Q^{2}$. b) Comparison of the $p_{T}$ spectra at two different $Q^{2}$ values (bins 2 and 7) for fixed (large) $W$. c) Comparison of the $p_{T}$ spectra at two different $Q^{2}$ values (bins 6 and 8 ) for fixed (large) $x$. d) Comparison of the $p_{T}$ spectra in two different $\eta$ intervals in the kinematic bin 3 (low $x$, intermediate $Q^{2}$ ). The mean values of $x$ and $Q^{2}$ are indicated in the figures. The error bars represent the quadratic sum of the statistical and systematic errors. 


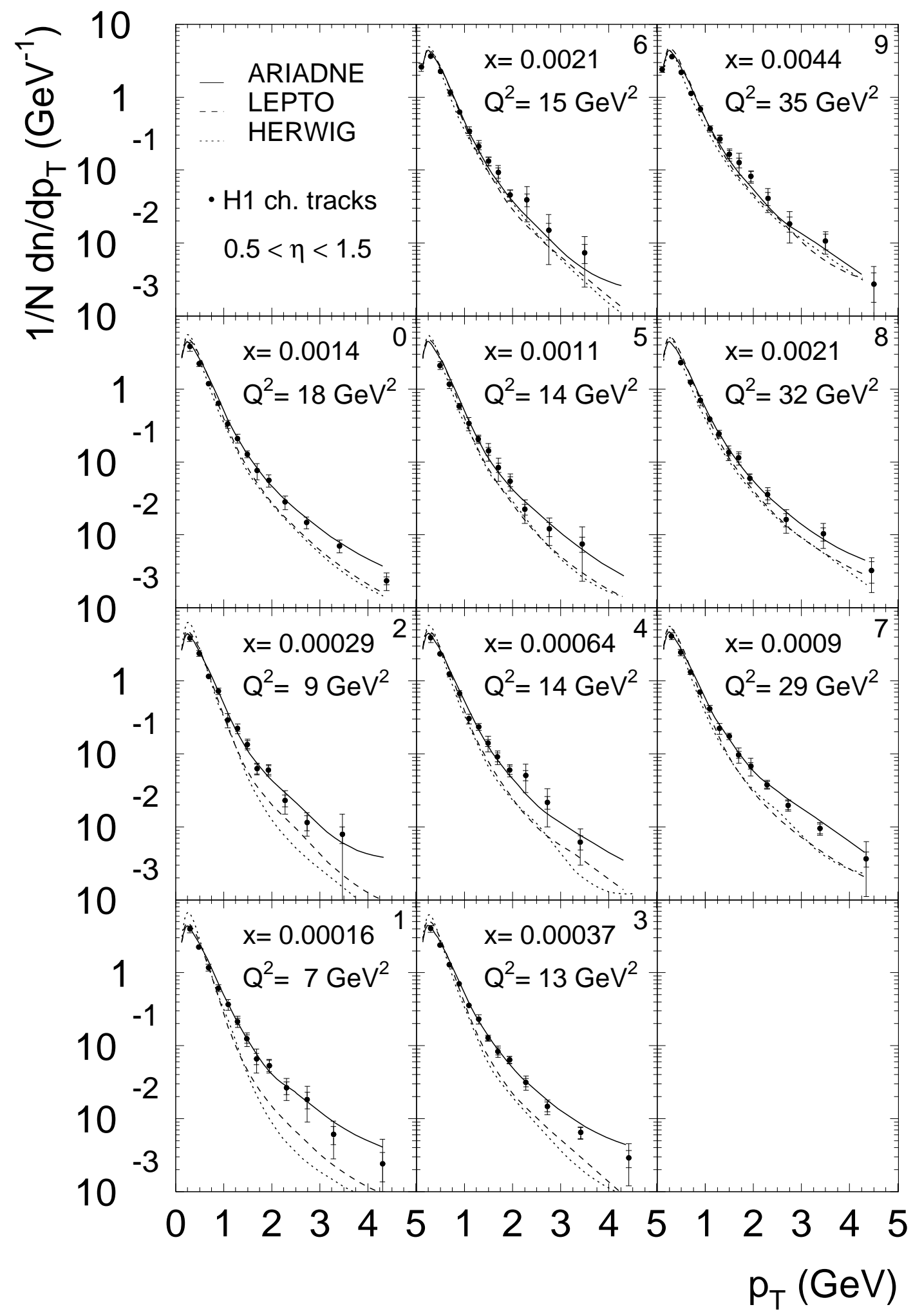

Figure 4: The transverse momentum spectra of charged particles, measured in the CMS in the pseudorapidity interval $0.5<\eta<1.5$. Data are shown for seven different kinematic bins (see Table 1) plus the combined sample (bin 0). For comparison, the models ARIADNE (full line), LEPTO (dashed) and HERWIG (dotted) are overlayed. The mean values of $x$ and $Q^{2}$ are indicated. The inner error bars represent the statistical errors, the outer error bars the quadratic sum of statistical and systematic errors. 


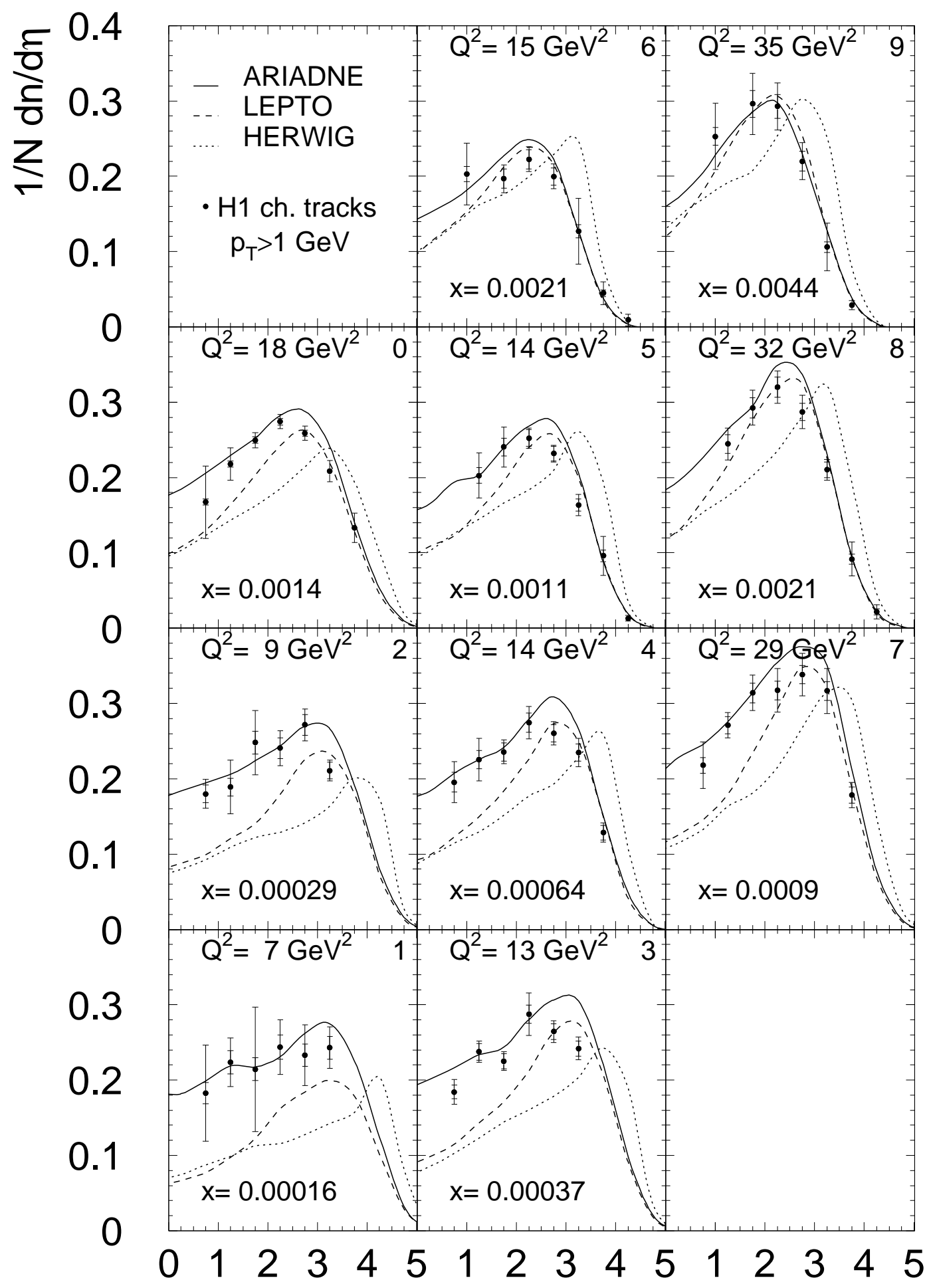

$\eta$

Figure 5: The CMS pseudorapidity spectra for charged particles with $p_{T}>1 \mathrm{GeV}$. The proton remnant direction is to the left. Data are shown for nine different kinematic bins (see Table 1) plus the combined kinematic region (bin 0). For comparison, the models ARIADNE (full line), LEPTO (dashed) and HERWIG (dotted) are overlayed. The mean values of $x$ and $Q^{2}$ are indicated. The inner error bars represent the statistical errors, the outer error bars the quadratic sum of statistical and systematic errors. 


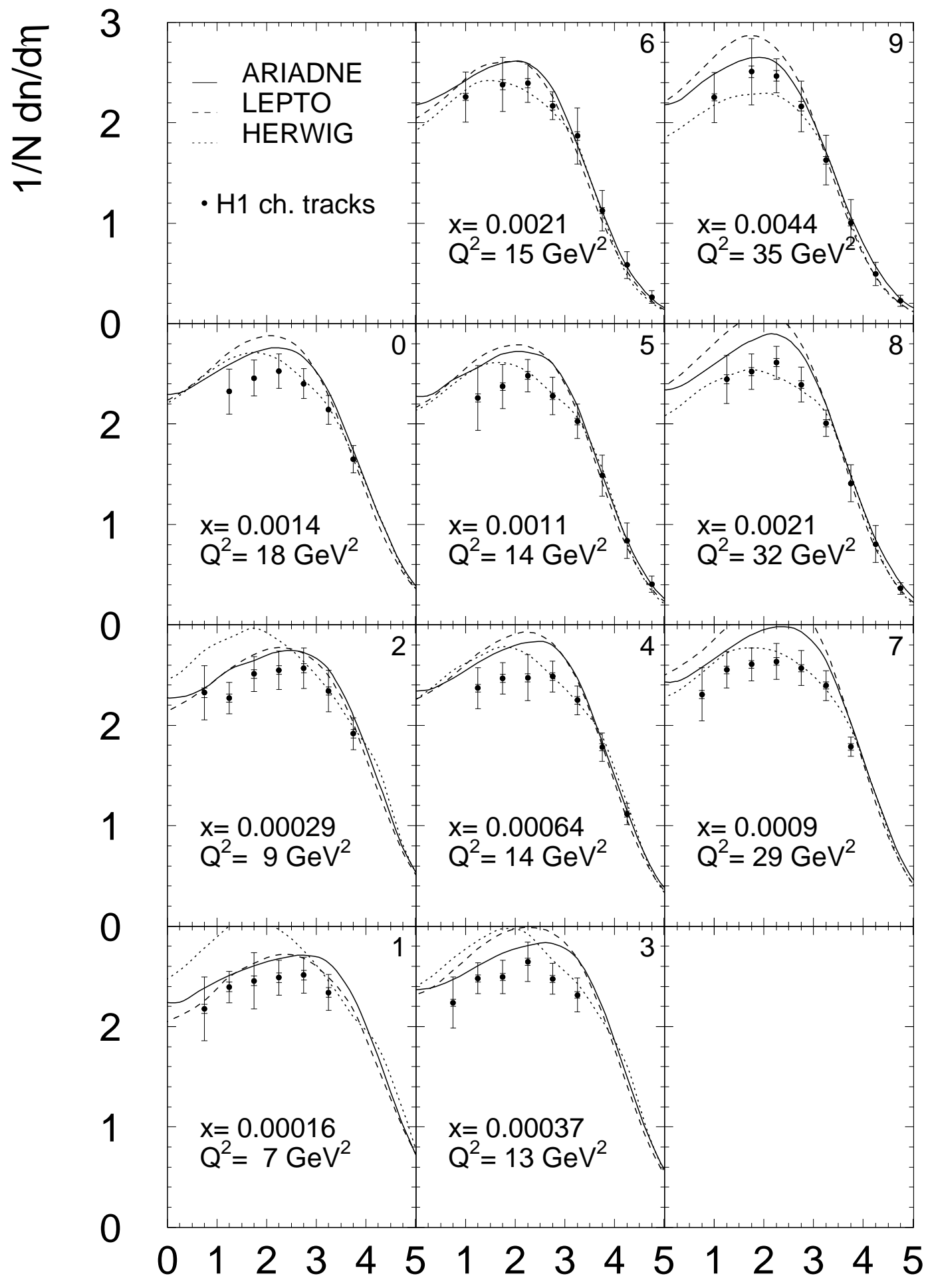

$\eta$

Figure 6: The CMS pseudorapidity spectra for all charged particles. The proton remnant direction is to the left. Data are shown for nine different kinematic bins (see Table 1) plus the combined kinematic region (bin 0). For comparison, the models ARIADNE (full line), LEPTO (dashed) and HERWIG (dotted) are overlayed. The mean values of $x$ and $Q^{2}$ are indicated. The inner error bars represent the statistical errors, the outer error bars the quadratic sum of statistical and systematic errors. 


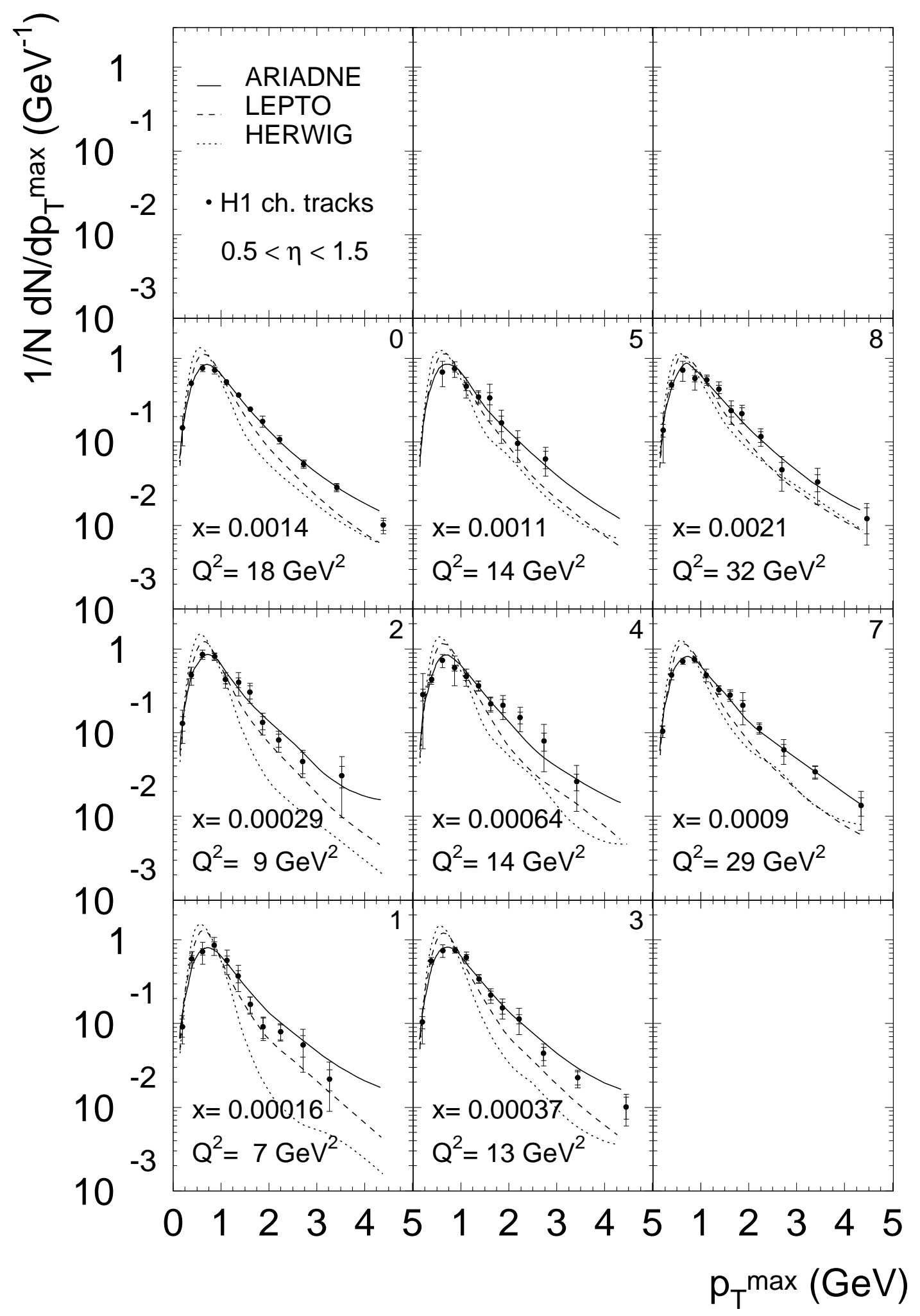

Figure 7: The $p_{T}^{\max }$ distribution as explained in the text, for the interval $0.5<\eta<1.5$. Data are shown for seven different kinematic bins (see Table 1) plus the combined kinematic region (bin 0). For comparison, the models ARIADNE (full line), LEPTO (dashed) and HERWIG (dotted) are overlayed. The mean values of $x$ and $Q^{2}$ are indicated. The inner error bars represent the statistical errors, the outer error bars the quadratic sum of statistical and systematic errors. 